\title{
LMMSE-Based Image Denoising in Nonsubsampled Contourlet Transform Domain
}

\author{
Md. Foisal Hossain ${ }^{1}$, Mohammad Reza Alsharif ${ }^{1}$, and Katsumi Yamashita ${ }^{2}$ \\ ${ }^{1}$ Department of Information Engineering, University of the Ryukyus, \\ Okinawa, Japan \\ foisalkuet@yahoo.com, asharif@ie.u.ryukyu.ac.jp \\ ${ }^{2}$ Graduate School of Engineering, Osaka Prefecture University, Osaka, Japan \\ Yamashita@eis.osakafu-u.ac.jp
}

\begin{abstract}
This paper proposes a nonsubsampled contourlet transform (NSCT) based multiscale linear minimum mean square-error estimation (LMMSE) scheme for image denoising. The contourlet transform is a new extension of the wavelet transform that provides a multi-resolution and multi-direction analysis for two dimension images. The NSCT expansion is composed of basis images oriented at various directions in multiple scales, with flexible aspect ratios. Given this rich set of basis images, the NSCT transform effectively captures smooth contours that are the dominant feature in natural images. To investigate the strong interscale dependencies of NSCT, we combine pixels at the same spatial location across scales as a vector and apply LMMSE to the vector. Experimental results show that the proposed approach outperforms wavelet method and contourlet based method both visually and in terns of the peak signal to noise ratio (PSNR) values at most cases.
\end{abstract}

Keywords: Denoising, NSCT, LMMSE, PSNR.

\section{Introduction}

An image is often corrupted by noise in its acquisition and transmission. Image denoising is used to remove the additive noise while retaining as much as possible the important signal features. In the recent years there has been a fair amount of research on wavelet thresholding and threshold selection for signal de-noising [1], [2]-[9]. However, wavelets are less effective for images where singularities are located both in space and directions.

Initially, the wavelet transform was considered to be a good decorrelator for images, and thus wavelet coefficients were assumed to be independent and were simply modeled using marginal statistics [10]. However, wavelet coefficients of natural images exhibit strong dependencies both across scales and between neighboring coefficients within a subband, especially around image edges. This gave rise to several successful joint statistical models in the wavelet domain [10]-[13].

One important feature of a transform is its stability with respect to shifts of the input signal. Shift invariance is very important in image denoising by thresholding because lack of shift invariance causes pseudo-Gibbs phenomena around singularities 
[7]. In addition to shift invariance, it is necessary that an efficient image representation should have the ability to capture geometrical structure exists in natural images. Contourlet transform [14] is a multidirectional and multiscale transform that is constructed by combining the Laplacian pyramid [15],[16] with directional filter bank (DFB), can be used to capture geometrical properties of images. However, due to downsamplers and upsamplers present in both the Laplacian pyramid and the DFB, the contourlet transform is not shift-invariant.

Hence, some new transforms have been introduced to solve this problem. Cunha and Do [17], developed nonsubsampled contourlet transform (NSCT), which is a fully shift-invariant version of contourlet transform, as well as multiscale, and multidirectional expansion. The NSCT is based on a nonsubsampled pyramid structure and nonsubsampled directional filter banks. NSCT transform allows for different and flexible number of directions at each scale, while achieving nearly critical sampling.

Although NSCT well decorrelates signals, strong interscale dependencies between NSCT coefficients still exist. Efficient modeling and exploiting such dependencies can significantly improve denoising performance. Denoising approach based on LMMSE considering interscale dependencies in wavelet domain was presented in [18]-[20]. However, the major drawback for wavelets in two-dimensions is their limited ability in capturing directional information.

In this paper, an LMMSE-based denoising approach with an interscale model is presented by using nonsubsampled contourlet transform (NSCT). NSCT do not have any downsampling in the decomposition and each NSCT subband has the same number of coefficients as the input image. We combine the NSCT coefficients with the same spatial location across adjacent scales as vector, to which LMMSE is then applied. Such an operation naturally incorporates the interscale dependencies of NSCT coefficients to improve the estimation. Experimental results show that the proposed approach outperforms other denoising methods in terms of visual quality.

The rest of the paper is organized as follows. Section 2 introduces NSCT in brief. The proposed LMMSE-based denoising approach is described in section 3 . Experimental results are presented in section 4. Finally a conclusion is presented in section 5.

\section{Construction of Nonsubsampled Contourlet Transform}

The NSCT is a fully shift-invariant, multi-scale, and multidirectional expansion that has a fast implementation. Overview of the NSCT is shown in Figure 1(a). The structure consists in a bank of filters that splits the 2-D frequency plane in the subbands shown in Figure 1(b). NSCT transform can thus be divided into two shiftinvariant parts which are as follow: a nonsubsampled pyramid structure that ensures the multi-scale property and a nonsubsampled DFB structure that gives directionality.

The contourlet transform is implemented via a two dimensional filter bank that decomposes an image into several directional subbands at multiple scales. To achieve this, the nonsubsampled contourlet transform is built upon nonsubsampled pyramids and nonsubsampled DFB [17]. First, a nonsubsampled pyramid split the input into a 
low pass subband and a high pass subband. Then a nonsubsampled DFB decomposes the high pass subband into several directional subbands. The scheme is iterated repeatedly on the low pass subband outputs of nonsubsampled pyramids.

The multiscale property of the NSCT is obtained from a shift invariant filtering structure that achieves subband decomposition similar to that of the Laplacian pyramid. The block diagram of the nonsubsampled pyramid is shown in Figure 2(a) and subband on the 2-D frequency plane is shown in Figure 2(b).

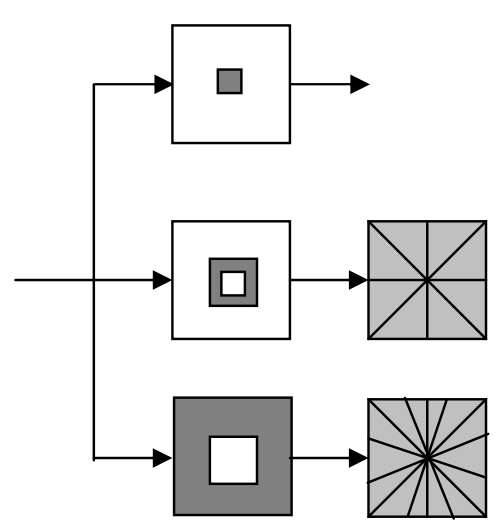

(a)

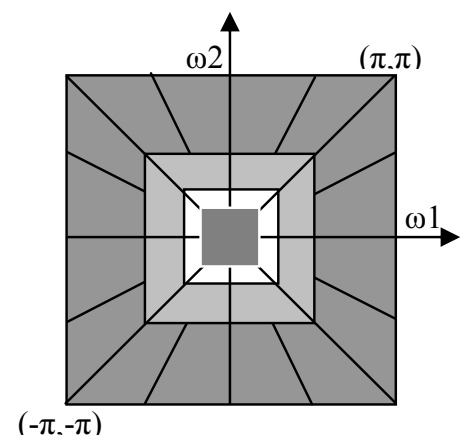

(b)

Fig. 1. Nonsubsampled contourlet transform. (a) NSFB structure that implements the NSCT. (b) Obtained idealized frequency partitioning.

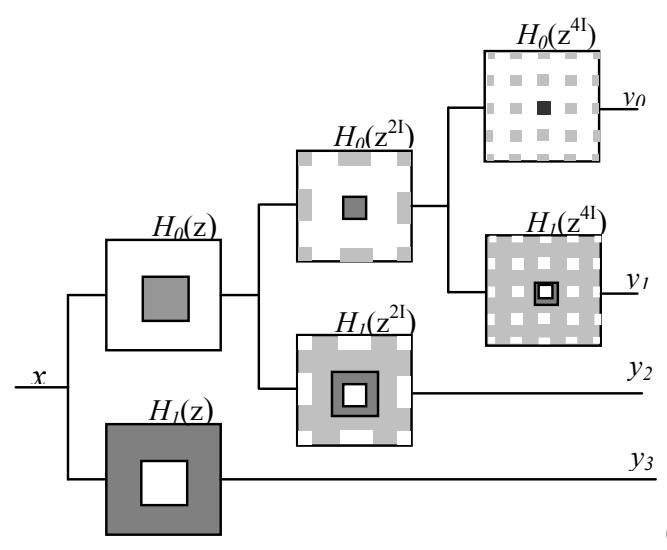

(a)

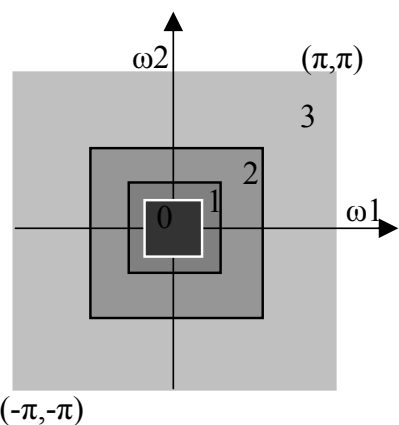

(b)

Fig. 2. Nonsubsampled pyramid is a 2-D multiresolution expansion. (a) Three stage pyramid decomposition. (b) Subbands on the 2-D frequency plane. 
Specifically, NSFB can be built from low-pass filter $H_{0}(\mathrm{z})$. Then we need to set

$$
H_{l}(\mathrm{z})=1-H_{0}(\mathrm{z}) \text {. }
$$

Perfect reconstruction condition is given as follows:

$$
H_{0}(z) G_{0}(z)+H_{1}(z) G_{1}(z)=1 .
$$

This condition is much easier to satisfy than the perfect reconstruction condition for critically sampled filter banks, and thus allows better filters to be designed.

The nonsubsampled DFB is a shift-invariant version of the critically sampled DFB in the contourlet transform. Nonsubsampled pyramids provide multiscale decomposition and nonsubsampled DFB's provide directional decomposition. The building block of a nonsubsampled DFB is also a two-channel nonsubsampled filter bank. However, shift invariance property is obtained by eliminating the downsamplers and upsamplers if the DFB.

\section{LMMSE Based Denoising}

NSCT is translation invariant and hence do not cause visual artifacts in threshold based denoising. For this the denoising scheme presented in this paper adopts NSCT. Suppose the original signal $f$ is corrupted with additive Gaussian white noise $\varepsilon$

$$
g=f+\varepsilon .
$$

where $\varepsilon \in N\left(0, \sigma^{2}\right)$. Applying NSCT to the noisy signal g, at scale j yields

$$
w_{j}=x_{j}+v_{j}
$$

where $w_{j}$ is coefficients at scale $j, x_{j}$ and $v_{j}$ are the expansions of $f$ and $\varepsilon$, respectively. Suppose the variance of $v_{j}$ is $\sigma_{j}^{2}$ and that of $x_{j}$ and $v_{j}$ are both zero mean, the LMMSE of $x_{j}$ is

$$
\hat{x}_{j}=c^{\prime} \cdot w_{j} .
$$

with

$$
c=\frac{\sigma_{x_{j}}^{2}}{\sigma_{x_{j}}^{2}+\sigma_{j}^{2}} .
$$

The factor $c$ is always less than 1, thus the magnitude of estimated NSCT coefficients would be less than that of $w_{j}$.

After the LMMSE result $\hat{\vec{x}}_{j}$ is obtained, only the component $\hat{x}_{j}$ is extracted. Estimation of $\hat{x}_{j+1}$ would be obtained form the LMMSE result $\hat{\bar{x}}_{j+1}$. 


\section{Experimental Results}

To evaluate the performance of the proposed approach this section compares the proposed scheme with other popular denoising schemes: wavelet based hardthresholding (WH), wavelet based soft-thresholding (WS). The three benchmark images named Lena, Barbara and Cameraman with dimension $512 \times 512$ are used for the experiments. The noisy images are obtained by adding Gaussian white noise to the noise free image.

Figure 3 and Figure 4 show the denoising performance of the above mentioned methods using the experimented images. From these figures, it can be observed that the proposed approach is better than the standard denoising methods.

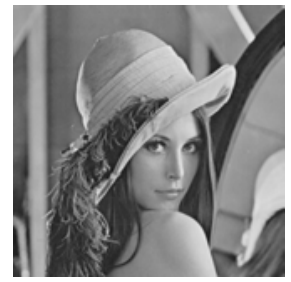

(a)

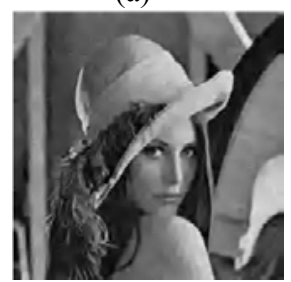

(e)

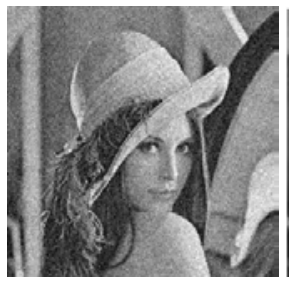

(b)

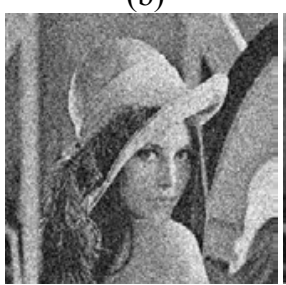

(f)

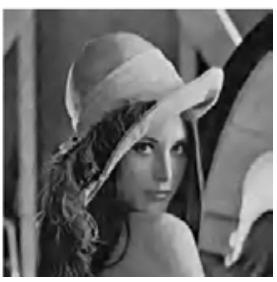

(c)

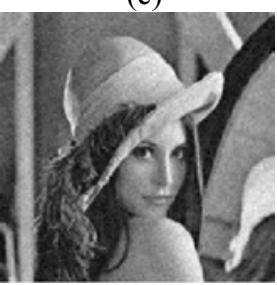

(g)

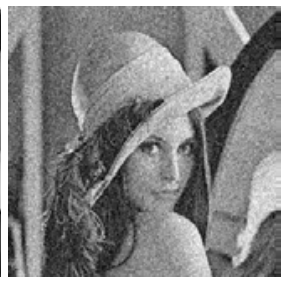

(d)

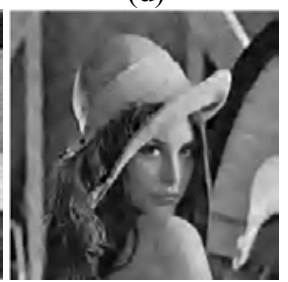

(h)

Fig. 3. Denoising results over Lena image (a) Original image with dimension $512 \times 512$; (b) noisy image $(\sigma=20)$; (c) denoised image over b; (d) noisy image $(\sigma=25)$; (e) denoised image over d; (f) noisy image $(\sigma=30)$; (g) denoised image using soft wavelet threshold over $\mathrm{f}$; (h) denoised image over $f$ using the proposed method

In order to evaluate the performance of the proposed method numerically, we measured peak signal to noise ration (PSNR), which is defined as:

$$
P S N R=10 \log _{10} \frac{I_{\max }^{2}}{\frac{1}{M N} \sum_{i, j}(X(i, j)-Y(i, j))^{2}} .
$$

where $I_{\max }$ is the maximum gray level value of the image, $X_{i, j}$ is the gray value of pixel at $(i, j)$ of the original image, $Y_{i, j}$ is the gray value of pixel at $(i, j)$ of the denoised image, and $M$ and $N$ are the numbers of row and column of the image respectively. 


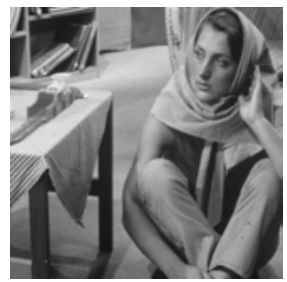

(a)

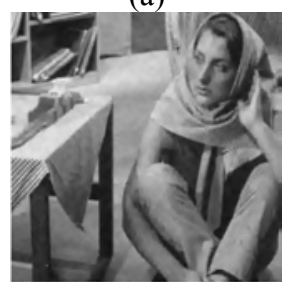

(e)

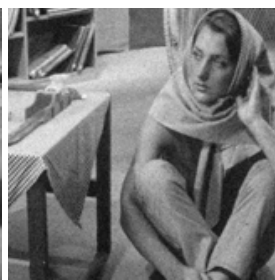

(b)

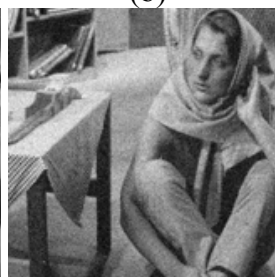

(f)

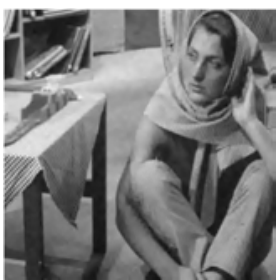

(c)

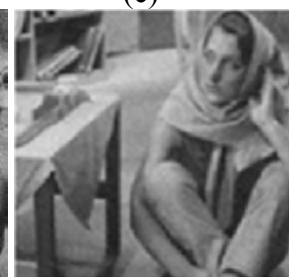

(g)

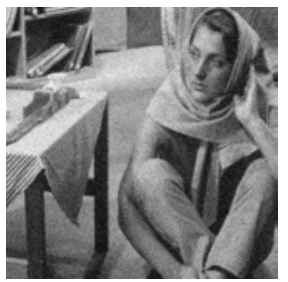

(d)

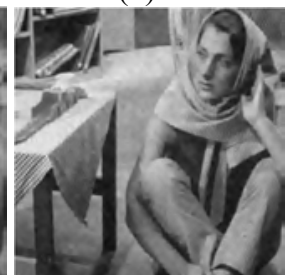

(h)

Fig. 4. Denoising results over Barbara image (a) Original image with dimension $512 \times 512$; (b) noisy image $(\sigma=20)$; (c) denoised image over b; (d) noisy image $(\sigma=25)$; (e) denoised image over d; (f) noisy image $(\sigma=30)$; (g) denoised image using soft wavelet threshold over $\mathrm{f}$; (h) denoised image over $f$ using the proposed method

Table 1. PSNR comparison of different methods over experimented images with different noise level

\begin{tabular}{ccccc}
\hline Image & $\begin{array}{c}\text { Noise } \\
\text { level }\end{array}$ & $\begin{array}{c}\text { Wavelet } \\
\text { hard threshold }\end{array}$ & $\begin{array}{c}\text { Wavelet } \\
\text { soft } \\
\text { threshold }\end{array}$ & $\begin{array}{c}\text { Proposed } \\
\text { method }\end{array}$ \\
\hline \multirow{3}{*}{ Lena } & 20 & 31.42 & 31.67 & 34.76 \\
& 25 & 30.71 & 30.76 & 32.87 \\
& 30 & 29.16 & 29.37 & 32.16 \\
\hline \multirow{3}{*}{ Cameraman } & 20 & 28.42 & 28.46 & 30.64 \\
& 25 & 27.22 & 27.29 & 29.12 \\
& 30 & 26.36 & 26.41 & 27.83 \\
\hline \multirow{3}{*}{ Barbara } & 20 & 23.26 & 23.72 & 27.57 \\
& 25 & 23.12 & 23.37 & 27.16 \\
& 30 & 22.36 & 22.47 & 26.73 \\
\hline
\end{tabular}

Table 1 list the PSNR results of the three methods on the experimented images corrupted by different levels of additive Gaussian noise. Observing the table, it can be grasped that the proposed approach outperforms other denoising methods.

\section{Conclusion}

In this paper, we have presented LMMSE-based denoising approach with NSCT interscale model. The NSCT is a fully shift-invariant, multi-scale, and 
multidirectional expansion. The NSCT coefficients at the same spatial locations at two adjacent scales are presented as a vector and LMMSE is applied to the vector. The NSCT interscale dependencies are thus exploited to improve the signal estimation. The proposed method gives highest PSNR values than other methods. It is ascertained from the experimental results that the proposed LMMSE based denoising approach outperforms other denoising methods.

\section{References}

1. Donoho, D.L.: De-Noising by Soft Thresholding. IEEE Trans. Info. Theory 43, 933-936 (1993)

2. Grace Chang, S., Yu, B., Vattereli, M.: Adaptive Wavelet Thresholding for Image Denoising and Compression. IEEE Trans. Image Processing 9, 1532-1546 (2000)

3. Donoho, D.L., Johnstone, I.M.: Adapting to Unknown Smoothness via Wavelet Shrinkage. Journal of American Statistical Assoc. 90(432), 1200-1224 (1995)

4. Grace Chang, S., Yu, B., Vattereli, M.: Wavelet Thresholding for Multiple Noisy Image Copies. IEEE Trans. Image Processing 9, 1631-1635 (2000)

5. Grace Chang, S., Yu, B., Vattereli, M.: Spatially Adaptive Wavelet Thresholding with Context Modeling for Image Denoising. IEEE Trans. Image Processing 9, 1522-1530 (2000)

6. Lang, M., Guo, H., Odegard, J.E.: Noise reduction Using Undecimated Discrete wavelet transform. IEEE Signal Processing Letters (1995)

7. Coifman, R.R., Donoho, L.D.: Translation Invariant De-noising. In: Antoniadis, A., Oppenheimm, G. (eds.) Wavelet and Statistics, pp. 125-150. Springer, Berlin (1995)

8. Bao, P., Zhang, L.: Noise Reduction for Magnetic Resonance Images via Adaptive multiscale Products Thresholding. IEEE Trans. Medical Imaging 22(9), 1089-1099 (2003)

9. Mallat, S.G.: A Theory for Multiresolution Signal Decomposition: the Wavelet Representation. IEEE Trans. on Pattern Analysis and Machine Intelligence 11(7), 674-693 (1989)

10. Romberg, J.K., Choi, H., Baraniuk, R.G.: Bayesian Tree-Structured Image Modeling Using Wavelet-Domain Hidden Markov Models. IEEE Trans. Image Proc. 10(7), 10561068 (2001)

11. Crouse, M., Nowak, R.D., Baraniuk, R.G.: Wavelet-Based Signal Processing Using Hidden Markov Models. IEEE Trans. Signal Proc. 46, 886-902 (1998)

12. Wainwright, M.J., Simoncelli, E.P., Willsky, A.S.: Random Cascades on Wavelet Trees and Their Use in Modeling and Analyzing Natural Images. Journal of Appl. and Comput. Harmonic Analysis 11, 89-123 (2001)

13. Do, M.N., Vetterli, M.: The contourlet transform: An efficient directional multiresolution image representation. IEEE Trans. Image Process. 14(12), 2091-2106 (2005)

14. Burt, P.J., Adelson, E.H.: The Laplacian pyramid as a compact image code. IEEE Trans. commun. 31(4), 532-540 (1983)

15. Do, M.N., Vetterli, M.: Framing pyramids. IEEE Trans. Signal Process. 51(9), 2329-2342 (2003)

16. Cunha, L., Zhou, J., Do, M.N.: The nonsubsampled contourlet transform: theory, design and applications. IEEE Trans. Image Proc. 15(10), 3089-3101 (2006) 
17. Mihçak, M.K., Kozintsev, I., Ramchandran, K., Moulin, P.: Low Complexity Image Denoising Based on Statistical Modeling of Wavelet Coefficients. IEEE Signal Process. Lett. 6(12), 300-303 (1999)

18. Li, X., Orchard, M.: Spatially Adaptive Image Denoising Under Overcomplete Expansion. In: Int. Conf. Image Process., Vancouver, Canada, pp. 300-303 (2000)

19. Zhang, L., Bao, P., Xiaolin, W.: Multiscale LMMSE-Based Image Denoising with Optimal Wavelet Selection. IEEE Trans. on Circuits and Systems for Video Technology 15(4), 469-481 (2005) 